Anaesthesist 2022 $\cdot 71: 233-242$

https://doi.org/10.1007/s00101-021-01051-1

Angenommen: 7. September 2021

Online publiziert: 18 . Oktober 2021

(c) Der/die Autor(en) 2021

\section{Volatile Anästhetika zur präklinischen Analgesie durch Rettungssanitäter - Eine Übersicht}

\author{
Helmut Trimmel ${ }^{1,2,3} \cdot$ Alexander Egger, ${ }^{3,4} \cdot$ Reinhard Doppler, ${ }^{3,5}$. \\ Christoph Beywinkler ${ }^{1,3} \cdot$ Wolfgang G. Voelcke ${ }^{3,6,7,8} \cdot$ Janett Kreutziger ${ }^{2,9}$ \\ ${ }^{1}$ Abteilung für Anästhesie, Notfall- und Allgemeine Intensivmedizin, Landesklinikum Wiener Neustadt, \\ Wiener Neustadt, Österreich; ${ }^{2}$ Karl Landsteiner Institut für Notfallmedizin, Wiener Neustadt, Österreich; \\ ${ }^{3}$ Christophorus Flugrettung, Österreichischer Automobil-, Motorrad- und Touring Club, ÖAMTC, Wien, \\ Österreich; ${ }^{4}$ Abteilung für Anästhesiologie und Intensivmedizin, Landesklinikum Scheibbs, Scheibbs, \\ Österreich; ${ }^{5}$ Abteilung für Innere Medizin, Landeskrankenhaus Rottenmann-Bad Aussee, Rottenmann, \\ Österreich; ${ }^{6}$ Abteilung für Anästhesiologie und Intensivmedizin, AUVA Unfallkrankenhaus Salzburg, \\ Salzburg, Österreich; ${ }^{7}$ Paracelsus Medizinische Privatuniversität, Salzburg, Österreich; ${ }^{8}$ Department für \\ Gesundheitsstudien, Universität Stavanger, Stavanger, Norwegen; ${ }^{9}$ Klinik für Anästhesie und \\ Intensivmedizin, Medizinische Universität Innsbruck, Innsbruck, Österreich
}

\title{
Zusammenfassung
}

Patienten mit Schmerzen können durch den nichtärztlichen Rettungsdienst mitunter nur inadäquat versorgt werden, da aufgrund rechtlicher Einschränkungen die Anwendung stark wirksamer Schmerzmittel (Opioide) bzw. ausbildungsbedingt eine i.v.-Therapie in Deutschland und Österreich oft nicht möglich ist. Häufig müssen Notärzte für schmerzgeplagte Patienten nachgefordert werden, wodurch deren Verfügbarkeit für z. B. vitale Notfälle reduziert sein kann. Inhalativ zu verabreichende Analgetika könnten hierfür eine interessante Alternative darstellen.

Derzeit steht dazu in Deutschland und Österreich Lachgas $\left(\mathrm{N}_{2} \mathrm{O}\right.$, als Livopan ${ }^{\circledR}$ im Handel) zur Verfügung, eine Mischung aus jeweils $50 \%$ Lachgas und Sauerstoff. In Österreich ist seit 2018 auch Methoxyfluran (Penthrop ${ }^{\circledR}$ ) zur Behandlung mäßiger bis starker Schmerzen nach einem Trauma für die prä- und innerklinische Anwendung bei Erwachsenen zugelassen.

In der Zusammenschau der vorhandenen Literatur, jahrzehntelanger Erfahrung in der Anwendung der Sauerstoff-Lachgas-Mischung im angloamerikanischen Bereich und von inhalativem Methoxyfluran v. a. in Australien sowie aktuellen Studien aus Europa kann gefolgert werden, dass diese bei Einhaltung der Anwendungsvorschriften effektiv, sicher und nebenwirkungsarm sind. Dies bestätigt auch eine eigene Untersuchung zu Methoxyfluran im präklinischen Einsatz. Die Anwendung von Lachgas ist aufgrund des Druckgaszylinders von der Handhabung her etwas aufwendig; Methoxyfluran ist einfacher anzuwenden und bei starken Schmerzen auch wirksamer. Die Zulassung von Methoxyfluran ist jedoch auf Erwachsene beschränkt, wo es mit zunehmendem Alter deutlich besser wirkt. Der Einsatz von Lachgas und insbesondere Methoxyfluran könnte aufgrund der Datenlage wie auch eigener Erfahrungen für rettungsdienstliches Fachpersonal nach entsprechender Einweisung empfohlen werden.

\section{Schlüsselwörter}

Inhalative Analgesie · Rettungsdienst · Methoxyfluran · Prähospitale Schmerztherapie . Rettungsdienstliches Fachpersonal 


\section{Hintergrund}

Die wirksame Behandlung akuter Schmerzen außerhalb eines Krankenhauses kann für nichtärztliches Rettungsdienstpersonal eine Herausforderung darstellen. Häufig müssen Notärzte für schmerzgeplagte $\mathrm{Pa}$ tienten nachgefordert werden, wodurch deren Verfügbarkeit für z. B. vitale Notfälle limitiert sein kann.

An möglichen Ursachen werden in der Literatur verschiedene Aspekte diskutiert:

- unzureichende Ausbildung bzw. klinische Erfahrung des eingesetzten Personals,

- fehlende Therapiestandards [3, 22],

- Sorge um potenzielle, ggf. nichtbeherrschbare Nebenwirkungen [22, 49],

- gesetzliche Limitationen für das nichtärztliche Rettungsdienstpersonal in Bezug auf die i.v.-Verabreichung von Medikamenten bzw. die Anwendung von Opioiden,

- unzureichende Erfassung (z. B. mittels Numeric Rating Scale [NRS] oder visueller Analogskala [VAS]) und Unterschätzung von Schmerzzuständen im notärztlichen wie auch im nichtärztlichen Bereich [22, 49],

- verzögerter Wirkungseintritt bzw. zu geringe Wirkung von Nicht-Opioidanalgetika bei starken Schmerzen, auch wenn einzelne Untersuchungen gegenteilige Effekte aufzeigen [15, 21, 37].

\section{Voraussetzungen für nichtärztlich verabreichte Analgesie}

Eine adäquate analgetische Versorgung von Notfallpatienten ist prähospital wie innerklinisch ein wichtiges Qualitätskriterium, flächendeckend und ggf. auch arztunabhängig standardisiert sicherzustellen [27]. Dies sollte mit klaren Algorithmen und einfach anzuwendenden, nebenwirkungsarmen Medikamenten auch erreichbar sein. Folgende Voraussetzungen müssen aus Sicht der Autoren dabei erfüllt sein: kein kritischer Einfluss auf Vitalfunktionen (Bewusstsein, Atmung, Kreislauf), milde, einfach beherrschbare $\mathrm{Ne}-$ benwirkungen ohne Potenzial der Patientengefährdung, einfache Applikation, rascher Wirkungseintritt, gute Steuerbarkeit, einfache Lagerungsfähigkeit (Temperaturstabilität), geringer Dokumentationsaufwand (nicht wie bei Opioiden), geringes Missbrauchspotenzial, akzeptable Kosten. Daneben sind vertretbarer Schulungsaufwand und akzeptable Arbeitsplatzbelastung und schließlich die Freigabe zur Applikation durch Rettungsdienstfachpersonal gemäß nationalen gesetzlichen Festlegungen, wie z. B. dem österreichischen Sanitätergesetz (Bundesgesetzblatt [BGBI.] I Nr. 30/2002) oder dem Notfallsanitätergesetz in Deutschland (BGBI. I S 1384/2014), erforderlich.

\section{Medikamente zur inhalativen Analgesie}

In der Europäischen Union sind aktuell zwei Medikamente, die per inhalationem verabreicht werden und diese Voraussetzungen erfüllen könnten, zugelassen: Lachgas $\left(\mathrm{N}_{2} \mathrm{O}\right)$ und Methoxyfluran.

Bei inhalativen Analgetika entfällt naturgemäß die Notwendigkeit eines i.v.Zugangs für die Schmerzbehandlung, zudem erfolgt die Verabreichung patientengesteuert: Je nach Schmerzniveau kann der Patient die Zufuhr jederzeit unterbrechen oder durch Erhöhung von Atemfrequenz bzw. Atemzugvolumen auch intensivieren. Dies erfordert selbstverständlich ein Minimum an Compliance und Sprachverständnis. Der Wirkungseintritt ist rasch, die Verträglichkeit gut - kritische Nebenwirkungen (anhaltende Bewusstseinstrübung, interventionsbedürftige Atem- oder Kreislaufdepression) sind für beide Substanzen in der prähospitalen Anwendung nicht beschrieben [17, 43, 44].

Die analgetische Potenz von Methoxyfluran scheint im Vergleich zu Opioiden nicht sehr stark: Circa ein Drittel der Potenz von Morphin [40] bzw. $3 \mathrm{ml}$ Methoxyfluran etwa vergleichbar mit ca. $25 \mu \mathrm{g}$ Fentanyl i.v. [36], wurde in limitierten Settings beschrieben. Trotzdem kann Methoxyfluran in der klinischen Anwendung auch mit Opioiden bestehen [35]. Im angloamerikanischen Raum hat sich die Mischung aus jeweils $50 \%$ Sauerstoff und Lachgas (Entonox ${ }^{\circledR}$ ) prähospital wie innerklinisch seit Jahrzehnten bewährt $[6,17]$ und wird auch in Kombination mit Opioiden angewendet $[28,41,50]$. Die Zulassung in Deutschland und Österreich zur "Behand- lung kurzzeitiger Schmerzzustände von leichter bis mittlerer Intensität" gemäß Fachinformation liegt für Patienten jeden Alters $>1$ Monat seit Jahren vor. Lachgas ist hier als Livopan ${ }^{\circledR}(\mathrm{Fa}$. Linde Healthcare, 85764 Oberschleißheim bzw. bzw. 1030 Wien, Österreich) im Handel.

Methoxyfluran ist ein volatiles Anästhetikum, welches nur mehr in niedriger, rein analgetischer Konzentration zum Einsatz kommt (in Australien seit 1975, in Neuseeland seit 2002, in einigen Ländern Osteuropas seit 2010) $[8,11,13,24]$. Die Anwendung von Methoxyfluran ist an mehr als 5 Mio. Patienten erfolgt und an über 200.000 erwachsenen und pädiatrischen Patienten in Registerdaten ( $\boldsymbol{\bullet}$ Tab. 1 ) oder prospektiven Studien untersucht $[9,34$, 40]. Ernste Nebenwirkungen, insbesondere das Auftreten einer malignen Hyperthermie, wurden bis dato nicht publiziert [32].

Während sich Lachgas in Mitteleuropa trotz einiger positiver Studien [18, 29] bislang kaum durchgesetzt hat, war Methoxyfluran hier bis vor Kurzem gar nicht zugelassen. Erst 2018 erhielt es die Zulassung durch die European Medicine Agency (EMA); in Österreich wird es unter dem Namen "Penthrop ${ }^{\circledR}$ “ zur Behandlung mittlerer und starker Schmerzen nach Trauma vertrieben. In Deutschland stoppte der Hersteller Mundipharma im Oktober 2019 die Markteinführung von Methoxyfluran („Penthrox ${ }^{\circledR}$ ). Als Gründe wurden gemäß einer Information der Arbeitsgemeinschaft Notärzte in Nordrhein-Westfalen (AGNNW) "Zulassungsund Anwendungsbeschränkungen" ohne weiterführende Informationen sowie „Probleme durch den Brexit" angegeben. Nach mündlicher Mitteilung seitens der Firma hatte die Entscheidung v.a. Marketinggründe, die ausschließlich Deutschland betrafen. Dabei hatten einige Rettungsdienste bereits die Einführung von Penthrox ${ }^{\circledR}$ geplant [1].

\section{Literaturüberblick}

\section{Lachgas}

\section{Pharmakologie}

Lachgas wurde erstmals 1772 von Priestley und Leeds synthetisiert, aber erst 1844 als Analgetikum praktisch eingesetzt. In 


\begin{tabular}{|c|c|c|c|c|c|}
\hline Autor & Typ & Setting & $\begin{array}{l}\text { Anzahl, Patien- } \\
\text { ten [Methoxyflu- } \\
\text { ran-Medikation] }\end{array}$ & Alter & Methoxyfluran, Ergebnis \\
\hline $\begin{array}{l}\text { Middleton } \\
2010[22]\end{array}$ & $\begin{array}{l}\text { Retrospektiv, } \\
\text { Vergleichsstu- } \\
\text { die }\end{array}$ & $\begin{array}{l}\text { Prähospital, } \\
\text { Australien }\end{array}$ & $42.844[19.235]$ & $\begin{array}{l}\text { 16-100 } \\
\text { Jahre }\end{array}$ & $\begin{array}{l}3,2 \text { mediane Reduktion in der VNRS-11 (59,1\% erreichten } \geq 30 \% \text { ige } \\
\text { Reduktion in der Schmerzwahrnehmung) }\end{array}$ \\
\hline $\begin{array}{l}\text { Johnston } \\
2011[23]\end{array}$ & $\begin{array}{l}\text { Retrospektiv, } \\
\text { Beobachtungs- } \\
\text { studie }\end{array}$ & $\begin{array}{l}\text { Prähospital, } \\
\text { Australien }\end{array}$ & $1024[465]$ & $\begin{array}{l}\text { Keine } \\
\text { Einschrän- } \\
\text { kung }\end{array}$ & VAS-Reduktion: 5 min: 2,0 ; bei Klinikeinlieferung: 2,5 \\
\hline $\begin{array}{l}\text { Oxer } 2007 \\
{[42]}\end{array}$ & $\begin{array}{l}\text { Retrospektiv, } \\
\text { Beobachtungs- } \\
\text { studie }\end{array}$ & $\begin{array}{l}\text { Prähospital, } \\
\text { Australien }\end{array}$ & $17.334[10.706]$ & $\begin{array}{l}\text { Keine } \\
\text { Einschrän- } \\
\text { kung }\end{array}$ & $>90 \%$ gute oder mind. teilweise Schmerzerleichterung \\
\hline $\begin{array}{l}\text { Bendall } \\
2011[18]\end{array}$ & $\begin{array}{l}\text { Retrospektiv, } \\
\text { Vergleichsstu- } \\
\text { die }\end{array}$ & $\begin{array}{l}\text { Prähospital, } \\
\text { Australien }\end{array}$ & 3312 [2093] & 5-15 Jahre & $\begin{array}{l}\text { Effektive Analgesie bei } 78,3 \% \text { aller Patienten, (VNRS-11-Reduktion } \\
\text { in } \geq 30 \% \text { ) }\end{array}$ \\
\hline $\begin{array}{l}\text { Jacobs } \\
2010[31]\end{array}$ & $\begin{array}{l}\text { Retrospektiv, } \\
\text { Vergleichsstu- } \\
\text { die }\end{array}$ & $\begin{array}{l}\text { Prähospital, } \\
\text { Australien }\end{array}$ & $135.770[17.629]$ & $\begin{array}{l}1-104 \\
\text { Jahre }\end{array}$ & $\begin{array}{l}\text { Kein Unterschied in kardialen Komplikationen, Nieren- oder Leber- } \\
\text { funktionseinschränkungen, Diabetes oder Krebserkrankungen bei } \\
\text { Patienten, die Methoxyfluran erhalten haben (Vgl. zu Gesamtkollek- } \\
\text { tiv) }\end{array}$ \\
\hline
\end{tabular}

der klinischen Anwendung ist die Farbund Geruchslosigkeit insbesondere bei Kindern vorteilhaft. Lachgas bewirkt über den N-Methyl-D-Aspartat(NMDA)-Rezeptor kaum Atemdepressionen, jedoch auch keine suffiziente Anästhesie (minimale alveoläre Konzentration, $M A C=110 \%$ ) [33]. Durch die hohe Diffusionskapazität flutet das Gas im Körper extrem schnell an und wieder ab. Bei Anwendung von Konzentrationen $>50 \%$ ist die Gabe von $100 \%$ igem Sauerstoff über mindestens $3 \mathrm{~min}$ am Ende der Verabreichung notwendig, um eine alveoläre Diffusionshypoxie zu vermeiden. Vorsicht ist weiters bei der notfallmedizinischen Anwendung bei Innenohrverletzungen oder Pneumothorax geboten. Eine leichte Sympathikusstimulation wurde ebenfalls beschrieben, die den gering negativ inotropen Effekt kompensieren kann. Bei kurzfristiger, prähospitaler Anwendung drohen außer Übelkeit, Bewusstseinseinengung und der erwähnten Diffusion in Hohlorgane aufgrund der geringen Metabolisierung (beachte: Darmbakterien) keine relevanten Nebenwirkungen. Die Gabe von Antiemetika in niedriger Dosierung, insbesondere $5 \mathrm{HT}_{3}$-Antagonisten oder Droperidol (1,25 mg/70 kgKG) kann evtl. erforderlich sein. Hochdosierte Anwendung über einen längeren Zeitraum ( $>6 \mathrm{~h}$ ) kann jedoch zu Störungen im Vitamin- $B_{12}$-Stoffwechsel führen (Oxidation des Kobaltions im Kobalamin).
Vitamin $\mathrm{B}_{12}$ ist ein wichtiger Kofaktor der Methioninsynthetase, die wiederum bei der Synthese von DNA, Cholin, Phospholipiden und Myelin mitwirkt. Bei Mangel an Cobalamin drohen daher hämatologische, neurologische und reproduktionstoxische Wirkungen sowie ggf. Störungen der embryonalen Entwicklung. Daher sollte es bei Patienten nach einer Gastrektomie, mit „Blind-loop"-Syndrom, Alkoholismus oder Patienten, die sich streng vegetarisch/vegan ernähren, aufgrund deren eingeschränkter Kobalaminreserven nur kurzfristig angewandt werden.

\section{Praxis}

Lachgas-Sauerstoff-Mischungen sind seit Jahrzehnten zur Analgesie im Einsatz. Die Literatur ist umfangreich, sowohl für den prä- als auch für den innerklinischen Bereich (Notfallaufnahme, Geburtshilfe, Pädiatrie). Beispielhaft und relativ aktuell sei hier die randomisierte, placebokontrollierte Studie von Ducasse et al. in Toulouse genannt, die die erfolgreiche Anwendung von Lachgas durch Paramedics bei erwachsenen Traumapatienten zeigen konnten. In zwei Drittel der Patienten mit einem initialen Schmerzniveau von 6 auf der NRS wurde eine ausreichende Analgesie (NRS $<3$ nach $15 \mathrm{~min}$ ) erreicht [18]. Auch bei Kindern und Jugendlichen wurde Lachgas, allerdings in Kombination mit intranasalem Fentanyl, für kurzfristige, notfallmedizinische Eingriffe wie Repositionierungen und Lagerungsmanöver erfolgreich eingesetzt [30]. Die Rate an Nebenwirkungen wie tiefere Sedierung oder Übelkeit und Erbrechen war mit $<5 \%$ für ein innerklinisches Setting tolerabel [51]. Auch in Deutschland wurde die Lachgas-Sauerstoff-Mischung bei erwachsenen Traumapatienten $(n=35)$, etwa im Rahmen der LabET-Studie, erfolgreich als Monotherapeutikum eingesetzt und gefolgert, dass dieses Medikament für die Anwendung durch nichtärztliches Rettungsdienstfachpersonal durchaus geeignet wäre [29].

In Österreich wurde Lachgas zur Überbrückung bei stärksten Schmerzen (im Schnitt NRS=8) 2012 in einem Pilotprojekt des Roten Kreuzes Steiermark durch nichtärztliches Rettungsdienstfachpersonal bis zum Eintreffen des Notarztes in einer kleinen Kohorte von erwachsenen Notfallpatienten $(n=65)$ nach einem Trauma angewandt. Die Schmerzintensität sank binnen 10 min nach Beginn der Applikation von initial 8 Punkten auf der NRSSkala um 3,4 Punkte. Diese Behandlung wurde von $91 \%$ der Patienten subjektiv als angenehm empfunden (ausreichende Schmerzreduktion), wenngleich ein NRS von 4,6 Punkten nach Therapie aus ärztlicher Sicht nicht zufriedenstellen kann. Ein Nachteil von Lachgas ist jedenfalls das relativ hohe Gewicht der notwendigen Devices - Druckgasflasche, Druckminderer und Demand-Ventil ( $\bullet$ Abb. 1) beanspruchen relativ viel Platz und lassen $\mathrm{N}_{2} \mathrm{O}$ für 


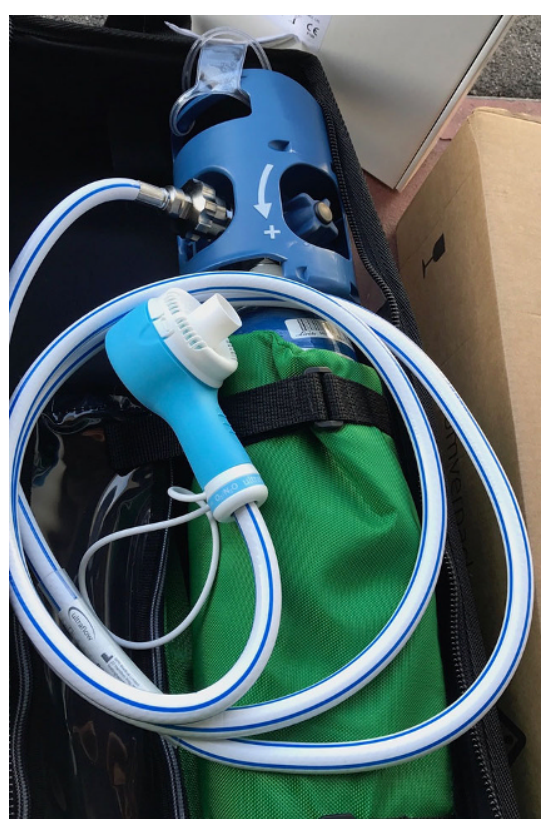

Abb. 1 ム Livopan ${ }^{\circledR}$. Druckgaszylinder mit Leitung und Demand-Ventil in einer Tragtasche zur prähospitalen Verabreichung von Lachgas

manche Bereiche (z. B. alpines Setting) als eher ungeeignet erscheinen.

\section{Methoxyfluran}

\section{Pharmakologie}

Bereits 1940 wurden fluorierte Kohlenwasserstoffe von Robbins auf ihre anästhesiologische Aktivität hin untersucht [48]. Doch erst 1956 testeten Van Poznak und Artusio 2,2-Dichlor-1:1-difluorethylmethylester $\left(\mathrm{C}_{3} \mathrm{H}_{4} \mathrm{Cl}_{2} \mathrm{~F}_{2} \mathrm{O}\right)$ aus der Klasse der Dialkylester in Hunden [45]. Am Menschen wurde Methoxyfluran erstmals 1960 untersucht $[5,46]$. Die klare, nahezu farblose Flüssigkeit mit charakteristisch fruchtigem Geruch (Fachinformation Penthrop ${ }^{\circledR}$ [26]) hat von allen bisher beschriebenen volatilen Anästhetika die höchste Potenz mit der niedrigsten MAC $(0,16 \%)$. In niedriger Dosierung hat es sehr gute analgetische Eigenschaften [52]. Die Metabolisierung findet in der Leber statt, wo es zu Fluorid und Dichloressigsäure demethylisiert (CYP450 2E14) wird, um dann durch Dechlorierung zu Methoxydifluoressigsäure und Oxalsäure abgebaut zu werden. Dabei wird Fluorid freigesetzt, was bei lang dauernder Anwendung in hohen Dosierungen nephrotoxisch wirken kann [16, 38]. Bei kurzer Anwendung im Rahmen von notfallmedizinischen Einsätzen oder
Interventionen ist diesbezüglich kein Risiko zu erwarten. Dennoch wird seitens des Herstellers empfohlen, Methoxyfluran bei anamnestischem Hinweis auf vorbestehende Leberschädigung oder klinisch manifester Niereninsuffizienz nicht einzusetzen. Als unerwünschte Wirkungen werden Schwindel, Kopfschmerzen, Schläfrigkeit, Mundtrockenheit und Übelkeit angegeben. Seltener, doch für den Rettungsdienst relevant sind euphorische Stimmungslage, ein Gefühl wie bei Trunkenheit, Dysarthrie, Amnesie, Diplopie, Blutdruckschwankungen ohne Interventionserfordernis (Hypo- und Hypertonie), Hustenreiz und Speichelsekretion. Bei richtiger Anwendung eines Penthrop ${ }^{\circledR}$ Inhalators mit Aktivkohlekammer zur Absorption des rückgeatmeten Methoxyflurans sollte kaum Methoxyfluran in die Umwelt gelangen. Bei chronischer Exposition, etwa in geschlossenen Räumen, wurde laut Fachinformation ein Anstieg von Leberenzymen, Blutharnstoff-Stickstoff und Serumharnsäure gefunden.

\section{Praxis}

In PubMed finden sich unter den Suchbegriffen "inhaled methoxyflurane" and „emergency“ aktuell 55 Studien. Das Medikament ist, wie erwähnt, seit Jahrzehnten im notfallmedizinischen prä- und innerklinischen Einsatz. Millionen von Patienten wurden durch Paramedics prähospital [13, 34,40 ] wie auch von Notfallmedizinern innerklinisch erfolgreich und sicher versorgt $[14,25,43]$. Bei über 200.000 Notfallpatienten mit kurzer Anwendung von Methoxyfluran, die in publizierte Studien eingeschlossen wurden, wurde bisher kein einziger Fall von maligner Hyperthermie - um die potenziell gefährlichste Nebenwirkung zu nennen - oder Hinweise auf Nierenschädigung dokumentiert. Auch sonst sind die Nebenwirkungen mild und es ist eigentlich erstaunlich, dass die Anwendung dieser Substanz in Europa bisher nur von begrenztem Interesse war. Mittlerweile liegen für Methoxyfluran neben der Zulassungsstudie (,STOP!-Trial“[14]) weitere europäische, prospektive, randomisierte Studien aus Frankreich, Italien und Spanien vor, die in innerklinischen Notfallaufnahmen durchgeführt wurden [12, 39, 47]. Methoxyfluran senkte im Vergleich zu Placebo Schmerzen signifikant - gemessen anhand der VAS (0-100) um durchschnittlich 35 Punkte [14]. In Spanien wurde Methoxyfluran bei posttraumatischen Schmerzen mit der lokal etablierten Standardanalgesie (NSAR bei VAS $\leq 6$, darüber ggf. zusätzliche Opioidgaben) multizentrisch verglichen und war dieser nach 20 min deutlich überlegen [12]. Daten aus Notaufnahmestationen in Italien zeigten ebenfalls, dass der analgetische Effekt von Methoxyfluran z.T. hochsignifikant der Standardtherapie überlegen war. Hier wurde Methoxyfluran bei 270 Erwachsenen nach Extremitätentrauma gegen i.v. verabreichtes Paracetamol, Ketoprofen (bei NRS 4-6) und/oder Morphin (bei NRS 7-10) getestet [39]. Auch in Frankreich wurde Methoxyfluran multizentrisch bei Erwachsenen nach Trauma innerklinisch gegen Placebo getestet: Je stärker die Ausgangsschmerzen waren, desto größer war der Effekt von Methoxyfluran. Mit dem inhalativen Analgetikum wurde deutlich weniger Zusatzmedikation in Form von Opioiden benötigt [47].

Nach diesen Erfolg versprechenden innerklinischen Anwendungen stellte sich natürlich die Frage nach der prähospitalen Anwendbarkeit im zentraleuropäischen, notfallmedizinischen Setting. Vor Kurzem wurde in Österreich dazu eine Anwendungsbeobachtung von Methoxyfluran zur prähospitalen Analgesie nach einem Trauma durchgeführt [53]. Methoxyfluran wurde an luft- als auch bodengebundenen Notarztmitteln zur Behandlung von mittleren bis starken Schmerzen bei erwachsenen, bewusstseinsklaren Patienten eingesetzt. Bedingt durch die SARS-CoV-2-Pandemie wurden zwar nur 109 der geplanten 200 Patienten eingeschlossen, dennoch konnten wichtige, z. T. auch signifikante Ergebnisse in Bezug auf Anwenderfreundlichkeit und Effizienz von Methoxyfluran detektiert werden. Die mittels NRS erhobene Schmerzstärke lag bei Eintreffen des Notarztes im Median bei 8 Punkten (7-8), 15 min nach Behandlungsbeginn bei 4 (3-5). Bei knapp zwei Drittel der Patienten wurde keinerlei zusätzliche Analgesie benötigt, wobei sich in der Wirksamkeit eine Altersabhängigkeit zeigte. Je älter die Patienten waren, umso effizienter war Methoxyfluran, d.h., desto seltener wurde eine Zusatzmedikation (bei über 80 -Jährigen nur $11 \%$ ) benötigt. Dies 


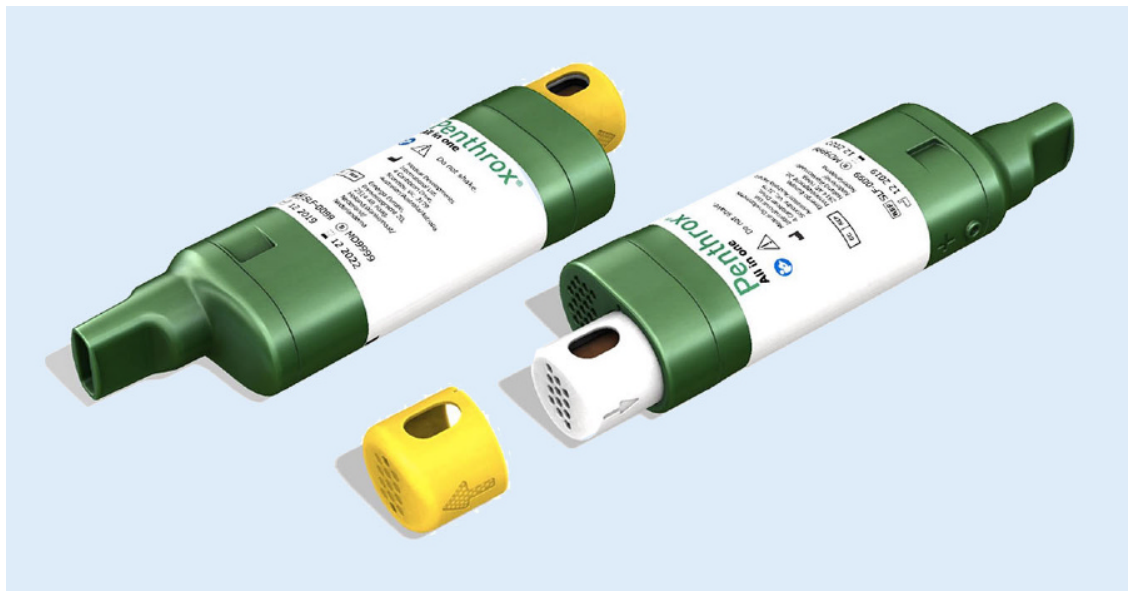

Abb. 2 ム Penthrox Inhaler "Selfie“. Der neue Inhalator soll die Anwendung von Penthrox ${ }^{\circledR}$ vereinfachen. (MitfreundlicherGenehmigung, all rights reserved $\odot$ Medical Developments International (MDI), Scoresby, Victoria, Australia)

lässt sich mit der Abhängigkeit des MACWerts vom Alter gut erklären und könnte sohin Anlass für Überlegungen zu Dosisvarianzen für verschiedene Altersgruppen geben. Waren Blutdruck und Herzfrequenz der Patienten zu Beginn der Behandlung schmerzbedingt etwas ausgelenkt, normalisierten sich diese unter der Behandlung; Atmung und Bewusstsein wurden nicht beeinträchtigt. Auch die Nebenwirkungen, die in etwa der Hälfte aller behandelten Patienten auftraten, waren in jedem Fall mild. Am häufigsten wurde ein Schwindelgefühl mit 37,8\% angegeben. Mit zunehmendem Alter nahmen die Nebenwirkungen tendenziell in ihrer Häufigkeit, nicht jedoch in der Schwere zu. Unangenehme Geruchs- bzw. Geschmacksempfindungen als Nebenwirkung gaben fünf Patienten an. Ein größeres Problem waren technische Schwierigkeiten: Bei $15 \%$ aller Anwendungen tropfte während der Vorbereitung des Inhalators Flüssigkeit aus dem Mundstück, was eine deutlich verstärkte Geruchsbelastung in der Umgebung zur Folge hatte. In geschlossenen Räumen (Hubschrauberkabine, Rettungswagen) wurde bei jedem fünften Einsatz eine Geruchsbelastung auch ohne das beschriebene Befüllungsproblem rückgemeldet. Dennoch wurde die Anwenderfreundlichkeit in der Summe von den Notärzten als gut bezeichnet und die Zufriedenheit von Patienten und Anwendern war sehr hoch.

\section{Diskussion}

Die Literatur zeigt, dass die inhalative Schmerztherapie nach einem Trauma wie auch bei prozeduralen Schmerzen mit Methoxyfluran ebenso wie mit Lachgas effizient und gut verträglich ist, auch wenn nicht für alle Patienten eine ausreichende Analgesie mit einem inhalativen Analgetikum als Monotherapeutikum erreicht werden kann. Sie zeichnet sich durch einen raschen Beginn der Analgesie aus und wird von den Patienten positiv bewertet $[32,43,44]$. Methoxyfluran ist in der Handhabung deutlich weniger aufwendig als Lachgas [24, 35, 44], wenngleich die Anwendung ebenfalls nicht ganz simpel ist. Der Umgang mit dem Inhalator muss schmerzgeplagten Patienten - insbesondere älteren - einfühlsam erklärt werden: in der Akutsituation ist das nicht immer einfach. Die Wirksamkeit hängt entscheidend davon ab, ob der Patient die Handhabung richtig verstanden hat und durchführt. Die Möglichkeit zur Konzentrationserhöhung durch Verschließen des "dilutor hole" sollte etwa erst nach einer Eingewöhnungsphase angesprochen werden. Patienten können den bei voller Konzentration anfänglich scharfen Geschmack als eher unangenehm empfinden und dann eine weitere Anwendung möglicherweise ablehnen. Nach fünf bis sechs tiefen Atemzügen tritt dieses Phänomen kaum mehr auf, die Analgesie hingegen setzt bereits ein. Auch das Vorbereiten des Inhalators ist nicht ganz einfach: die Flüssigkeit kann beim Befüllungsvorgang aus dem Mundstück auf den Boden tropfen. Dies kann die Wirkung abschwächen bzw. die Nutzungsdauer verkürzen. Hier soll eine Neukonfektion des Devices Verbesserung bringen (- Abb. 2: "Penthrox ${ }^{\circledR}$ Inhaler Selfie", Medical Developments International Limited [MDI], Scoresby, Victoria, Australia). Der neue Inhalator wird die Wirkstoffphiole bereits integriert haben, sodass ein manuelles Befüllen nicht mehr erforderlich ist.

Negative Auswirkungen für das Personal sind im prähospitalen Einsatz weder für Lachgas noch für Methoxyfluran zu erwarten. Die gesetzlichen Grenzwerte für Lachgas sind in den Technischen Regeln für Gefahrstoffe (TRGS) in Deutschland durch die Bundesanstalt für Arbeitsschutz und Arbeitsmedizin [7] bzw. das Bundesministerium für Arbeit in Österreich [10] mit $100 \mathrm{ppm}$ als Tagesmittelwert festgelegt. Kurzfristige höhere Konzentrationen bis 400 ppm werden akzeptiert. Für Methoxyfluran werden in Deutschland und Österreich 2 ppm als Tagesmittelwert (bzw. $14 \mathrm{mg} / \mathrm{m}^{3}$ ) und $4 \mathrm{ppm}$ als kurzfristige Belastung in der Umgebungsluft als Grenzwert festgelegt $[2,10]$. Eine aktuelle Studie aus Frankreich, die körpernahe Konzentrationen von Methoxyfluran bei Pflegepersonal einer innerklinischen Notfallstation mit regelmäßiger Anwendung während der $8 \mathrm{~h}$-Schichten analysierte, fand eine mit 0,017 ppm sehr niedrige Durchschnittsbelastung [19]. Auch Messungen der Konzentration von Methoxyfluran in mobilen Ambulanzen konnte keine bedenklichen Konzentrationen beim behandelnden, nichtärztlichen Personal nachweisen $(<0,2 \mathrm{ppm})$. Die Schwelle für eine Geruchswahrnehmung von Methoxyfluran liegt weit unter den gesetzlichen Grenzwerten, aber über der durchschnittlichen Konzentration in Rettungsfahrzeugen und Ambulanzen (0,13-0,19 ppm) [4, 20]. In der Summe lässt sich trotz des wahrnehmbaren Geruchs von Methoxyfluran bzw. der nichtwahrnehmbaren Luftkonzentration von Lachgas selbst bei regelmäßigem Gebrauch als Notfallanalgetikum keine gesundheitliche Gefährdung der Behandler ableiten.

Die Bereitschaft von Notärzten, ein inhalatives Analgetikum einzusetzen, ist angesichts moderner, intranasal oder bukkal 


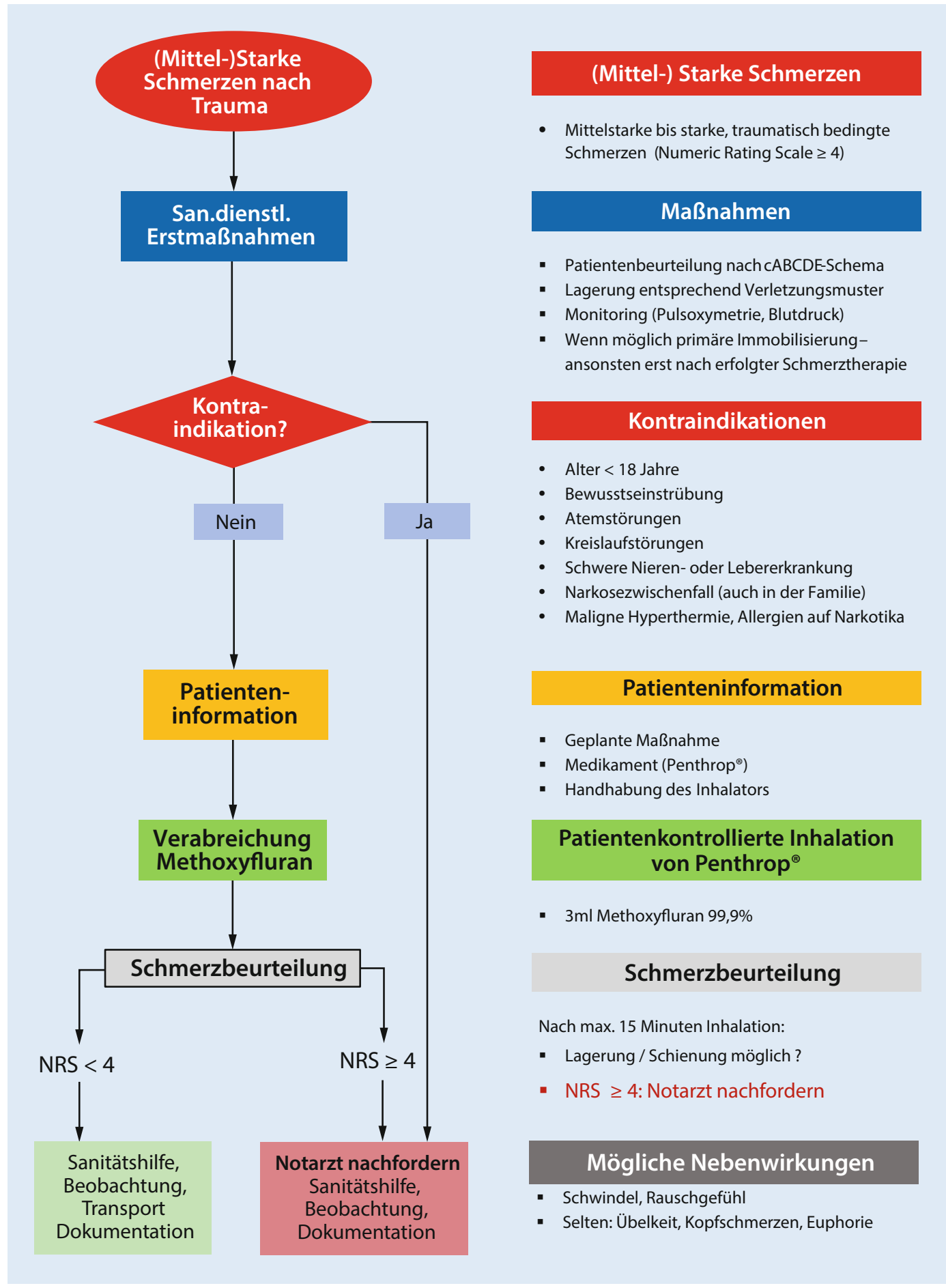

Abb. $3 \triangleleft$ Algorithmus zur Anwendung von Penthrop ${ }^{\circledR}$ durch nichtärztliches Personal im Rettungsdienst. Beispiel eines Algorithmus; die Nachforderung des notärztlichen Dienstes ist bei nichtausreichender Analgesie (NRS $\geq 4$ ) vorgesehen

appliziert rasch wirksamer Opioide wohl limitiert. Natürlich ist Methoxyfluran weniger wirksam als diese [40]; dieser Umstand muss jedoch gegen die aufwendigere Logistik, das Erfordernis einer exakten ärztlichen (!) Verabreichungsdokumentation, die strenge Überwachung der Lagerhaltung sowie v.a. gesetzliche Limitierungen der Anwendung von Opioiden durch nicht- ärztliche Berufsgruppen abgewogen werden. Notärzten stehen potente(re) Analgetika zur Verfügung: Unsere Ergebnisse wie auch die vorhandene Literatur bestätigen jedoch, dass Methoxyfluran auch rettungsdienstlichem Fachpersonal in Europa eine suffiziente und v.a. sichere Behandlung von Patienten mit mittleren bis starken Schmerzen ermöglichen kann; ggf. auch in Kombination mit i.v. zu verabreichenden Nichtopioidanalgetika. Unter Anwendung eines definierten Behandlungsalgorithmus (• Abb. 3) ließe sich die Zahl notärztlicher Einsätze ausschließlich zur Analgesie verringern und deren Verfügbarkeit für andere, vitalgefährdete Patienten erhöhen [23]. 


\section{Fazit für die Praxis}

- Lachgas und Methoxyfluran sind sicher anzuwendende, gut wirksame Inhalationsanalgetika. Lachgas ist für alle Altersgruppen, Methoxyfluran derzeit nur für erwachsene Patienten zugelassen, wobei ältere Patienten in der aktuellen Einheitsdosierung deutlich stärker von seiner analgetischen Wirkung profitieren.

- Die Applikation beider Medikamente erfolgt patientengesteuert; die unerwünschten Arzneimittelwirkungen bei kurzer Anwendung sind mild. Besondere Vorteile von Methoxyfluran liegen in der einfachen Logistik und stärkeren Analgesie; Limitationen in der Anwendung können aufgrund unzureichender Compliance auftreten. Ein weiterer Nachteil ist die Geruchsbelastung für das Rettungsdienstpersonal in geschlossenen Räumen, auch wenn dies nie die gesetzlich zulässigen Arbeitsplatzkonzentrationen erreicht. Hier wird herstellerseitig Abhilfe durch einen neuen Inhalator versprochen.

- Die Anwendung von Methoxyfluran anhand eines definierten Algorithmus kann aus unserer Sicht auch für nichtärztliches Personal empfohlen werden.

\section{Korrespondenzadresse}

\section{Prim. PD Dr. Helmut Trimmel, MSc}

Abteilung für Anästhesie, Notfall- und Allgemeine Intensivmedizin, Landesklinikum Wiener Neustadt

Corvinusring 3-5, 2700 Wiener Neustadt, Österreich

Helmut.Trimmel@wienerneustadt.lknoe.at

\section{Einhaltung ethischer Richtlinien}

Interessenkonflikt. H. Trimmel: grants, payments, support for travel from Mundipharma Austria: the „MetAir" trial was supported by an unrestricted grant from Mundipharma, Austria. H. Trimmel participated in advisory board meetings for methoxyflurane (Penthrox ${ }^{\circledR}$ ) in Vienna (2018) and London (2019), received travel reimbursement and honoraria for lectures in context with inhaled analgesia. Leadership or fiduciary role in ÖGARI: Helmut Trimmel chairs the section "Emergency Medicine" of ÖGARI. R. Doppler, A. Egger, C. Beywinkler, W.G. Voelckel und J. Kreutziger geben an, dass kein Interessenkonflikt besteht.

Für diesen Beitrag wurden von den Autoren keine Studien an Menschen oder Tieren durchgeführt. Für die aufgeführten Studien gelten die jeweils dort angegebenen ethischen Richtlinien.

Open Access. Dieser Artikel wird unter der Creative Commons Namensnennung 4.0 International Lizenz veröffentlicht, welche die Nutzung, Vervielfältigung, Bearbeitung, Verbreitung und Wiedergabe in jeglichem Medium und Format erlaubt, sofern Sie den/die ursprünglichen Autor(en) und die Quelle ordnungsgemäß nennen, einen Link zur Creative Commons Lizenz beifügen und angeben, ob Änderungen vorgenommen wurden.

Die in diesem Artikel enthaltenen Bilder und sonstiges Drittmaterial unterliegen ebenfalls der genannten Creative Commons Lizenz, sofern sich aus der Abbildungslegende nichts anderes ergibt. Sofern das betreffende Material nicht unter der genannten Creative Commons Lizenz steht und die betreffende Handlung nicht nach gesetzlichen Vorschriften erlaubt ist, ist für die oben aufgeführten Weiterverwendungen des Materials die Einwilligung des jeweiligen Rechteinhabers einzuholen.

Weitere Details zur Lizenz entnehmen Sie bitte der Lizenzinformation auf http://creativecommons.org/ licenses/by/4.0/deed.de.

\section{Literatur}

1. AGNNW (2019) Penthrox: Einführung auf den deutschen Markt durch Hersteller gestoppt. https://www.agnnw.de/?p=4443. Zugegriffen: 16 . März 2021

2. Ausschuß für Gefahrstoffe (AGS) (2004) Grenzwerte in der Luft am Arbeitsplatz. https://www. umwelt-online.de/recht/t_regeln/trgs/trgs900/ 900y2000.htm. Zugegriffen: 16. März 2021

3. Albrecht E, Taffe P, Yersin B et al (2013) Undertreatment of acute pain (oligoanalgesia) and medical practice variation in prehospital analgesia of adult trauma patients: a $10 \mathrm{yr}$ retrospective study. $\mathrm{Br}$ J Anaesth 110:96-106. https://doi.org/10.1093/ bja/aes355

4. Allison SJ, Docherty PD, Pons D, Chase JG (2011) Exposure to methoxyflurane: low-dose analgesia and occupational exposure. https://ajp. paramedics.org/index.php/ajp/article/view/712. Zugegriffen: 16. März 2021

5. Artusio JF, Poznak AV, Hunt RE et al (1960) A clinical evaluation of methoxyflurane in man. Anesthesiology 21:512-517. https://doi.org/10. 1097/00000542-196009000-00009

6. BaskettPJ (1970) Use of entonox in the ambulance service. BMJ 2:41-43. https://doi.org/10.1136/ bmj.2.5700.41

7. Ausschuss für Gefahrstoffe (AGS) (2027) $\mathrm{Ar}$ beitsplatzgrenzwerte. https://www.baua.de/ DE/Angebote/Rechtstexte-und-TechnischeRegeln/Regelwerk/TRGS/pdf/TRGS-900.pdf? blob=publicationFile\&v=18. Zugegriffen: 16. März 2021

8. Bendall JC, Simpson PM, Middleton PM (2011) Prehospital analgesia in New South Wales, Australia. Prehosp Disaster med 26:422-426. https://doi.org/10.1017/s1049023×12000180

9. Bendall JC, Simpson PM, Middleton PM (2011) Effectiveness of prehospital morphine, fentanyl, and methoxyflurane in pediatric patients. Prehosp Emerg Care 15:158-165. https://doi.org/10.3109/ 10903127.2010.541980

10. BGBI (2011) MAK-Werte und TRK-Werte. https://www.ris.bka.gv.at/Dokumente/BgblAuth/ BGBLA_2007_II_243/COO_2026_100_2_366060. pdfsig.Zugegriffen: 16. März 2021

11. Blair HA, Frampton JE (2016) Methoxyflurane: a review in trauma pain. Clin Drug Investig 36:1067-1073. https://doi.org/10.1007/s40261016-0473-0

12. Borobia AM, Collado SG, Cardona CC et al (2020) Inhaled methoxyflurane provides greater analgesia and faster onset of action versus standard analgesia in patients with trauma pain: inMEDIATE: a randomized controlled trial in emergency departments. Ann Emerg Med 75:315-328. https:// doi.org/10.1016/j.annemergmed.2019.07.028

13. Buntine P, Thom O, Babl F et al (2007) Prehospital analgesia in adults using inhaled methoxyflurane. Emerg Med Australas 19:509-514. https://doi.org/ 10.1111/j.1742-6723.2007.01017.x

14. Coffey F, Wright J, Hartshorn S et al (2014) STOP!: a randomised, double-blind, placebo-controlled study of the efficacy and safety of methoxyflurane for the treatment of acute pain. Emerg Med J 31:613-618. https://doi.org/10.1136/emermed2013-202909

15. Craig M, Jeavons R, Probert J, Benger J (2012) Randomised comparison of intravenous paracetamol and intravenous morphine for acute traumatic limb pain in the emergency department. Emerg Med J 29:37-39. https://doi.org/10.1136/emj. 2010.104687

16. Dayan A (2016) Analgesic use of inhaled methoxyflurane. Hum Exp Toxicol 35:91-100. https:// doi.org/10.1177/0960327115578743

17. Donen N, Tweed WA, White D et al (1982) Prehospital analgesia with entonox. Can Anaesth Soc J 29:275-279. https://doi.org/10.1007/bf03007131

18. Ducassé J-L, Siksik G, Durand-Béchu Met al (2013) Nitrous oxide for early analgesia in the emergency setting: a randomized, double-blind multicenter prehospital trial. Acad Emerg Med 20:178-184. https://doi.org/10.1111/acem.12072

19. Frangos J, Belbachir A, Dautheville $S$ et al (2020) Non-interventional study evaluating exposure to inhaled, low-dose methoxyflurane experienced by hospital emergency department personnel in France. BMJ Open 10:e34647. https://doi.org/10. 1136/bmjopen-2019-034647

20. Frangos J, Mikkonen A, Down C (2016) Derivation of an occupational exposure limit for an inhalation analgesic methoxyflurane (Penthrox ${ }^{\circledR}$ ). Regul Toxicol Pharmacol 80:210-225. https://doi.org/10. 1016/j.yrtph.2016.05.012

21. Friday JH, Kanegaye JT, McCaslin I et al (2009) Ibuprofen provides analgesia equivalent to acetaminophen-codeine in the treatment of acute pain in children with extremity injuries: a randomized clinical trial. Acad Emerg Med 16:711-716. https://doi.org/10.1111/j.1553-2712. 2009.00471.x

22. Galinski M, Hoffman L, Bregeaud D et al (2018) Procedural sedation and analgesia in trauma patients in an out-of-hospital emergency setting: a prospective multicenter observational study. Prehosp Emerg Care 22:497-505. https://doi.org/ 10.1080/10903127.2017.1413464

23. Galinski M, Ruscev M, Gonzalez G et al (2010) Prevalence and management of acute pain in prehospital emergency medicine. Prehosp Emerg Care 14:334-339. https://doi.org/10.3109/ 10903121003760218

24. Gaskell AL, Jephcott CG, Smithells JR, Sleigh JW (2016) Self-administered methoxyflurane for procedural analgesia: experience in a tertiary Australasian centre. Anaesthesia 71:417-423. https://doi.org/10.1111/anae.13377

25. Gillis M, Keirens A, Steinkamm C et al (2008) The use of methoxyflurane (penthrox) in the emergency department:411. Reg Anesth Pain Med 33:e247.1-e247. https://doi.org/10.1136/rapm00115550-200809001-00480

26. Grindlay J, Babl FE (2009) Review article: efficacy and safety of methoxyflurane analgesia in the emergency department and prehospital setting. Emerg Med Australas 21:4-11. https://doi.org/10. 1111/j.1742-6723.2009.01153.x 
27. Häske D, Schempf B, Gaier G, Niederberger C (2014) Prehospital analgesia performed by paramedics: quality in processes and effects under medical supervision. Anaesthesist 63:209-216. https://doi. org/10.1007/s00101-014-2301-3

28. Heinrich M, Menzel C, Hoffmann Fet al (2015) Selfadministered procedural analgesia using nitrous oxide/oxygen (50:50) in the pediatric surgery emergency room: effectiveness and limitations. Eur J Pediatr Surg 25:250-256. https://doi.org/10. 1055/s-0034-1371716

29. Hengefeld N, Lukas RP, Klaus S, Van Aken H, Boh A (2014) Lachgas-Sauerstoff-Gemisch (Livopan ${ }^{\circledR}$ ) bei Extremitätentrauma - die LAbET-Studie. Anasth Intensivmed 2:13

30. Hoeffe J, Trottier ED, Bailey B et al (2017) Intranasal fentanyl and inhaled nitrous oxide for fracture reduction: the FAN observational study. Am J Emerg Med 35:710-715. https://doi.org/10.1016/ j.ajem.2017.01.004

31. Jacobs IG (2010) Health Effects of Patients Given Methoxyflurane in the Pre-Hospital Setting: A Data Linkage Study. Open Emerg Med J 3:7-13. https:// doi.org/10.2174/1876542401003010007

32. Jephcott C, Grummet J, Nguyen N, Spruyt O (2018) A review of the safety and efficacy of inhaled methoxyflurane as an analgesic for outpatient procedures. Br J Anaesth 120:1040-1048. https:// doi.org/10.1016/j.bja.2018.01.011

33. Jevtović-Todorović $V$, Todorovć SM, Mennerick Set al (1998) Nitrous oxide (laughing gas) is an NMDA antagonist, neuroprotectant and neurotoxin. Nat Med 4:460-463. https://doi.org/10.1038/ nm0498-460

34. Johnston S, Wilkes GJ, Thompson JA et al (2011) Inhaled methoxyflurane and intranasal fentanyl for prehospital management of visceral pain in an Australian ambulance service. Emerg Med J 28:57-63. https://doi.org/10.1136/emj. 2009.078717

35. Kinsella J, Glavin R, Reid WH (1988) Patient-controlled analgesia for burn patients: a preliminary report. Burns Incl Therm Inj 14:500-503. https:// doi.org/10.1016/s0305-4179(88)80011-1

36. Lenz H, Høiseth LØ, Comelon M et al (2021) Determination of equi-analgesic doses of inhaled methoxyflurane versus intravenous fentanyl using the cold pressor test in volunteers: a randomised, double-blinded, placebo-controlled crossover study. Br J Anaesth 126:1038-1045. https://doi. org/10.1016/j.bja.2020.12.045

37. Luiz T, Scherer G, Wickenkamp A et al (2015) Prehospital analgesia by paramedics in RhinelandPalatinate: feasability, analgesic effectiveness and safety of intravenous paracetamol. Anaesthesist 64:927-936. https://doi.org/10.1007/s00101015-0089-4

38. Mazze RI, Raja SN (2006) Methoxyflurane revisited. Anesthesiology 105:843-846. https://doi.org/10. 1097/00000542-200610000-00031

39. Mercadante S, Voza A, Serra S et al (2019) Analgesic efficacy, practicality and safety of inhaled methoxyflurane versus standard analgesic treatment for acute trauma pain in the emergency setting: a randomised, open-label, active-controlled, multicentre trial in Italy (MEDITA). Adv Ther 36:3030-3046. https://doi.org/10.1007/s12325019-01055-9

40. Middleton PM, Simpson PM, Sinclair G et al (2010) Effectiveness of morphine, fentanyl, and methoxyfluranein the prehospital setting. Prehosp Emerg Care 14:439-447. https://doi.org/10.3109/ 10903127.2010.497896

\section{Volatile anesthetics for prehospital analgesia by paramedics-An overview}

Treatment of acute pain is a central task in emergency medicine. Yet, prehospital pain relief is often insufficient or delayed since the administration of potent intravenous analgesic drugs (such as opioids) is mostly limited to physicians due to legal restrictions or training deficiencies in Germany and Austria. Frequently, prehospitally operating emergency physicians have to be demanded later for anguished patients limiting disposability of physicians for patients who are in a potentially life-threatening condition. Thus, inhaled analgesics could represent an interesting alternative. A mixture of $50 \%$ nitrous oxide and $50 \%$ oxygen $\left(\mathrm{N}_{2} \mathrm{O}\right.$, Livopan $\left.{ }^{\circledR}\right)$ has been available in Germany and Austria for several years; however, prehospital use of Livopan has been merely realized and only one trial has been published. In addition, methoxyflurane $\left(\right.$ Penthrop $\left.{ }^{\circledR}\right)$, a volatile anesthetic from the group of the dialkyl esters (2-dichloro-1:1difluoroethyl-methyl-ester) was approved for the treatment of moderate to severe pain following trauma in adults in many European countries in recent years and was brought onto the market in Austria in 2018. Several in-hospital trials demonstrated high effectiveness in this setting.

This article discusses the effects and prehospital areas of application of both substances in the light of the existing literature. We provide a narrative overview of the current study situation and report on a recently performed prehospital application study of methoxyflurane (Penthrop ${ }^{\circledR}$ ) from Austria.

The need for pressurized gas cylinders for the use of $\mathrm{N}_{2} \mathrm{O}$ represents a certain limitation in prehospital use. Furthermore, in certain injuries such as of the inner ear or a pneumothorax $\mathrm{N}_{2} \mathrm{O}$ should not be used and the risk of diffusion hypoxemia has to be addressed. Users should be particularly careful and limit the use in alcohol addicts and vegans. The advances of $\mathrm{N}_{2} \mathrm{O}$ are that it is odorless, has a fast onset of action, the usability in patients over 1 month old and has stabilizing effects on the circulation. Plenty of literature regarding prehospital as well as in-hospital use of nitrous oxide in emergency, obstetric and pediatric settings show its effectiveness as a single drug as well as in combination with other analgesics, such as paracetamol or various opioids. Its long tradition in Anglo-American countries is also based on its safety and low rate of side effects.

Methoxyflurane is easier to store and handle and may be slightly more effective in severe pain after trauma; however, its approval is restricted to adults, where it works significantly better with increasing age, based on the declining minimal alveolar concentration (MAC) of all inhaled anesthetics with increasing age. Furthermore, decades of use of inhaled methoxyflurane in Australia have shown the drug is effective, safe and low in side effects and has a broad spectrum of applications. The use of methoxyflurane is limited in patients with severe hepatic or renal insufficiency and the characteristic odor has been described as unpleasant by some patients. In Europe, three large in-hospital trials showed strong pain relief in trauma patients, even comparable to opioids.

Overall, based on the current evidence, the use of nitrous oxide and even more of methoxyflurane may be recommended also for prehospital use by skilled paramedics.

\section{Keywords}

Inhaled analgesics - Methoxyflurane - Emergency medical service - Prehospital analgesia . Paramedic

41. Míguez MC, Ferrero C, Rivas A et al (2019) Retrospective comparison of intranasal fentanyl and inhaled nitrous oxide to intravenous ketamine and midazolam for painful orthopedic procedures in a pediatric emergency department. Pediatr Emer Care. https://doi.org/10.1097/pec. 0000000000001788
42. Oxer HF, Wilkes G (2007) Methoxyflurane is a safe, easy, effective analgesic for prehospital pain relief. Prehosp Disast Med 22:s77

43. Porter KM, Dayan AD, Dickerson S, Middleton PM (2018) The role of inhaled methoxyflurane in acute pain management. Open Access Emerg Med 10:149-164. https://doi.org/10.2147/oaem. s181222 
44. Porter KM, Siddiqui MK, Sharma I et al (2018) Management of trauma pain in the emergency setting: low-dose methoxyflurane or nitrous oxide? A systematic review and indirect treatment comparison. J Pain Res 11:11-21. https://doi.org/ 10.2147/jpr.s150600

45. Poznak AV, Artusio JF (1960) Anesthetic properties of a series of fluorinated compounds I. Fluorinated hydrocarbons. Toxicol Appl Pharmacol 2:363-373. https://doi.org/10.1016/0041-008x(60)90002-8

46. Poznak AV, Ray BS, Artusio JF (1960) Methoxyflurane as an anesthetic for neurological surgery. J Neurosurg 17:477-479. https://doi.org/10.3171/ jns.1960.17.3.0477

47. Ricard-Hibon A, Lecoules N, Savary D et al (2020) Inhaled methoxyflurane for the management of trauma related pain in patients admitted to hospital emergency departments: a randomised, double-blind placebo-controlled trial (PenASAP study). Eur J Emerg Med 27:414-421. https://doi. org/10.1097/mej.0000000000000686

48. Robbins BH (1940) Preliminary studies of anestheticactivity of luorinated hydrocarbons.JPharmaco Exp Ther 86:197-204

49. Scholten AC, Berben SAA, Westmaas AH et al (2015) Pain management in trauma patients in (pre)hospital based emergency care: current practice versus new guideline. Injury 46:798-806. https://doi.org/10.1016/j.injury.2014.10.045

50. Seiler M, Landolt MA, Staubli G (2019) Nitrous oxide $70 \%$ for procedural analgosedation in a pediatric emergency department-with orwithoutintranasal fentanyl? Pediatr Emer Care 35:755-759. https:// doi.org/10.1097/pec.0000000000001213

51. Seith RW, Theophilos T, Babl FE (2012) Intranasal fentanyl and high-concentration inhaled nitrous oxide for procedural sedation: a prospective observational pilot study of adverse events and depth of sedation. Acad Emerg Med 19:31-36. https://doi.org/10.1111/j.1553-2712.2011.01241. $\mathrm{x}$

52. Tomi K, MashimoT, Tashiro Cetal (1993) Alterations in pain threshold and psychomotor response associated with subanaesthetic concentrations of inhalation anaesthetics in humans. Br J Anaesth 70:684-686.https://doi.org/10.1093/bja/70.6.684

53. Trimmel H, Egger A, Doppler R et al Usability and efficacy of inhaled methoxyflurane for prehospital analgesia - a prospective, observational study. BMCEmerg Med

\section{Wann wirkt ein Smartphone-Foto ästhetisch?}

Social Media, Messenger-Dienste, Nachrichten und Werbung fluten uns täglich mit einer großen Menge von Bildern. Aber nicht alle geteilten Bilder werden als schön empfunden, während andere uns in ihren Bann ziehen. Aber warum ist das eigentlich so? Wodurch wird ein Foto zu einem großartigen Bild?

"Neben grundlegenden Bildmerkmalen legen wir den Fokus dabei vor allem auf die Person, die das Smartphones nutzt, sowie den Kontext, in dem ein Foto entsteht und geteilt wird", erklärt Helmut Leder von der Univerisät Wien, der gemeinsam mit Matthew Pelowski die wissenschaftliche Leitung des Projekts hält. Je nachdem ob man ein Selfie, ein Foto einer Bergtour, einer Städtereise oder vom letzten Restaurantbesuch betrachtet, können ganz unterschiedliche Aspekte mitbestimmen, ob das Bild als ästhetisch wahrgenommen wird oder nicht.

Ziel des Projekts ist es, die wahrgenommene Schönheit von Bildern in Abhängigkeit dieser verschiedenartigen Einflüsse zu untersuchen.

Als Grundlage dafür haben die Forscher*innen ein theoretisches Modell der Bildästhetik bei der SmartphoneFotografie entwickelt. Jede Betrachtung eines Bilds löst eine Abfolge von kognitiven Verarbeitungsprozessen aus. Innerhalb von Sekundenbruchteilen erfasst die Wahrnehmung wesentliche Aspekte eines Fotos. Dazu zählen einerseits ganz simple Bildmerkmale wie Farbe, Kontrast oder Beleuchtung. Aber auch Aspekte der Bildkomposition, wie etwa Symmetrie, Komplexität oder Bildausschnitt, bewirken schon einen ersten Eindruck. All diese Einflüsse führen bereits zu einer unmittelbaren ästhetischen Vorliebe für bestimmte Bilder, ohne dass darüber besonders nachgedacht wird.

Wenn man Bilder allerdings ein paar Sekunden länger betrachtet, kommen bereits höhere Denkprozesse ins Spiel. Das sind etwa Erinnerungen, die die Betrachter*innen mit dem Bild verbinden oder eine bestimmte Bedeutung, die Menschen dem Bildinhalt zuschreiben. Wir prüfen auch, ob das Bild zu unseren eigenen Vorstellungen und Erwartungen passt oder wie gut es der Situation entspricht, in der es aufgenommen wurde.

Das neue Modell ist eine erste systematische
Zusammenstellung all dieser Variablen, die die Schönheit solcher Bilder bestimmen. Dabei werden die zeitliche Abfolge von schnellem, spontanem Wischen bis hin zu ästhetischem Genuss - und besonders Unterschiede zwischen Personen miteinbezogen.

Momentan führt das Wiener Forschungsteam großangelegte Online-Experimente durch, um besser zu verstehen, wie die typischen Smartphone-Nutzer*innen die Entscheidung fällen, welche Bilder sie schön finden und mit anderen teilen möchten. "Wir möchten dabei vor allem die Wechselbeziehungen zwischen Inhalt, Bildgestaltung, aber auch kulturellem Kontext und Persönlichkeit verstehen. Diese wurden in der empirischen Forschung zur Wahrnehmung von Fotografien bislang weitgehend außer Acht gelassen", sagt Chris Valuch, Postdoc im Projekt.

Das Projekt fällt in den Forschungsschwerpunkt »Psychologie der Ästhetik«, der über den Cognitive Science Hub der Universität Wien auch starke interdisziplinäre Verbindungen zur Kunstgeschichte, der Biologie, den Künsten und den Neurowissenschaften pflegt. Dabei kooperiert das Team mit Ingenieur*innen von Huawei Technologies Finland. Das Projekt erweitert das Forschungsprogramm der Empirical Visual Aesthetics Labs von Helmut Leder und Matthew Pelowski, welche unter anderem durch Mittel aus dem EU Horizon 2020 Programm, dem Wiener Wissenschafts-, Forschungs- und Technologiefonds sowie dem Wissenschaftsfonds FWF gefördert werden.

Originalpublikation: Leder, Hakala, Peltoketo, Valuch \& Pelowski (2022). Swipes and Saves: A taxonomy of factors influencing aesthetic assessments and perceived beauty of mobile phone photographs. Frontiers in Psychology. DOI: https://doi.org/10.3389/fpsyg.2022.786977

Quelle: Universität Wien (www.medienportal.univie.ac.at [01.03.2022]) 
Hier steht eine Anzeige.

黑 Springer 